\section{Impact of variations in fatty liver on sonographic detection of focal hepatic lesions originally identified by CT}

\author{
Size $\mathrm{Wu}^{1}$, Rong Tu${ }^{2}$, Ruixia Nan', Guangqing Liu' ${ }^{1}$ Xiaojing Cui ${ }^{1}$, Xian Liang ${ }^{1}$ \\ Departments of ${ }^{1}$ Ultrasound and ${ }^{2}$ Radiology, Affiliated Hospital of Hainan Medical College, \\ Haikou, China
}

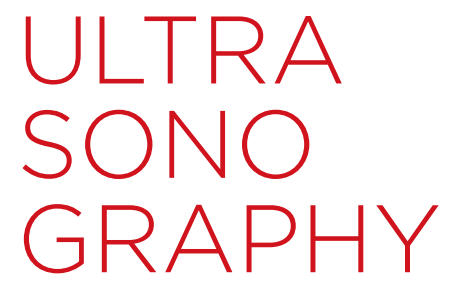

ORIGINAL ARTICLE

http://dx.doi.org/10.14366/usg.15022 pISSN: 2288-5919 - elSSN: 2288-5943 Ultrasonography 2016:35:39-46

Received: April 7, 2015

Revised: June 24, 2015

Accepted: June 26, 2015

Correspondence to:

Size Wu, MD, Department of Ultrasound, Affiliated Hospital of Hainan Medical College, No. 31, Longhua Road, Haikou 570102, China

Tel. +86-0898-66774347

Fax. +86-0898-66508833

E-mail:wsz074@aliyun.com

liver (162/177) vs. liver without fat infiltration (389/413) ( $P=0.277)$; mild fatty liver (162/177) vs. moderate fatty liver (190/212) ( $P=0.604)$; mild fatty liver (162/177) vs. severe fatty liver (83/112) $(\mathrm{P}<0.001)$; moderate fatty liver (190/212) vs. liver without fat infiltration (389/413) $(\mathrm{P}=0.051)$; moderate fatty liver $(190 / 212)$ vs. severe fatty liver $(83 / 112)(P<0.001)$; severe fatty liver $(83 / 112)$ vs. liver without fat infiltration (389/413) $(\mathrm{P}<0.001)$; and fatty liver $(435 / 501)$ vs. liver without fat infiltration $(389 / 413)(P<0.001)$.

Conclusion: Mild and moderate fatty liver are not significantly associated with the visualization of the lesion, while severe fatty liver usually impairs the detection of focal lesions in the liver. If a patient with severe fatty liver is suspected to have a liver tumor, ultrasonography should only be chosen cautiously in case of a missed diagnosis.

Keywords: Fatty liver; Liver neoplasms; Ultrasonography

\title{
Introduction
}

Ultrasonography (US) and computed tomography (CT) are widely used for liver focal lesion screening, follow-up, or further evaluation. Together with magnetic resonance imaging (MRI), they play an important role in current clinical medicine. US examination is often the first-line modality for liver evaluation, and findings of US involve subsequent management in asymptomatic cases. A primary aim of US examination is to determine whether there is a space-occupying lesion or not, regardless of its

This is an Open Access article distributed under the terms of the Creative Commons Attribution NonCommercial License (http://creativecommons.org/ licenses/by-nc/3.0/) which permits unrestricted noncommercial use, distribution, and reproduction in any medium, provided the original work is properly cited.

Copyright $(2016$ Korean Society of Ultrasound in Medicine (KSUM)

How to cite this article:

Wu S, Tu R, Nan R, Liu G, Cui X, Liang X. Impact of variations in fatty liver on sonographic detection of focal hepatic lesions originally identified by CT. Ultrasonography. 2016 Jan; 35(1):39-46. 
nature. However, the effect of US evaluation of the liver is subject to several factors, such as the operator's experience, the interference of gas, obesity, cirrhosis, fatty liver, and iron deposition [1-7]. Focal lesions may frequently be concurrent with fatty liver, for fatty liver (including non-alcohol fatty liver disease and liver fat infiltration from other causes) is common in the general population [8-10]. The presence of fatty liver may affect US imaging to some extent because focal lesions in fatty liver often show different features on imaging than those in a liver without fat infiltration $[3,4,7,8]$, which sometimes results in the downgrading of a US diagnosis $[4,7]$ but may not always impair visualization of tumors in the liver [3]. To our knowledge, no investigation has been conducted on the impact of different degrees of fatty liver on the US detection rate of focal liver lesions. The purpose of this study was to investigate the relationship of different degrees of fatty liver on the US detection of focal liver lesions.

\section{Materials and Methods}

\section{Design, Setting, and Subject Selection}

The Institutional Review Board of our affiliation approved this study and informed consent was waived due to the retrospective study design.

A study on the US detection rate of focal liver lesions in fatty liver was conducted from January 2010 through December 2014 in our hospital. Thirty thousand four hundred and twelve patients underwent US; among them 68 patients underwent unenhanced abdominal $\mathrm{CT}$ or combined chest and abdominal unenhanced $\mathrm{CT}$; 195 patients underwent enhanced abdominal CT or combined chest and abdominal $\mathrm{CT}$ without precontrast $\mathrm{CT} ; 2,027$ patients underwent abdominal CT/enhanced CT or combined chest and abdominal CT/ enhanced CT. Among the 2,027 patients who underwent both precontrast and contrast-enhanced $C T$, 1,839 patients were found with liver focal lesions. In the end, 1,241 patients with liver focal lesions were included in the study population, while 598 patients with glycogen storage disease, splenectomy, hepatic iron deposition, large lesions of diameter more than $9.0 \mathrm{~cm}$ and tiny lesions of diameter less than $0.5 \mathrm{~cm}$ were excluded; in addition, patients with more than 5 lesions in the liver were excluded due to the difficulty of accurately counting the number of lesions or absence of a concrete number in the records. The patients with focal liver lesions along with different degrees of fatty liver were categorized into groups with severe, moderate, and mild fatty liver. At first, 56 cases of severe and 92 cases of moderate fatty liver were all enrolled as group I; then 81 more cases of mild fatty liver were added to group I by selection with reference to a randomized table from the 308 cases of mild fatty liver. A subset of the patients with focal liver lesions but without fatty liver were selected with reference to a randomized table to be part of group II. In this way, 229 of 456 patients with fatty liver and focal liver lesions (134 males, 95 females; mean age, 36 years; range, 13 to 78 years) were selected as group I, and 200 of 785 patients with focal liver lesions and without fatty liver (119 males, 81 females; mean age, 33 years; range, 11 to 86 years) were selected as group II. Fig. 1 illustrates the flow chart of the study population and sample selection.

All procedures followed were in accordance with the ethical standards of the responsible committees on human experimentation (institutional and national) and with the Helsinki Declaration of 1975, as revised in 2008.

\section{US Study}

The US imaging study was performed by a sonologist (S.W.) with 15 years of experience, using an Aloka ProSound $\alpha 10$ (Aloka Co. Ltd., Mitaka, Tokyo, Japan) and Mindray DC-8 (Mindray Medical International Ltd., Senzheng, China), with 5- to 2-MHz convex transducers.

\section{CT Study}

The CT scans were performed using 64-MDCT (LightSpeed VCT, GE Healthcare, Milwaukee, WI, USA) scanners. For the contrastenhanced portions of the examinations, the patients received approximately $80-130 \mathrm{~mL}$ of iohexol $(300 \mathrm{mgl} / \mathrm{mL}$; Yangtze River Pharmaceutical Co. Ltd., Jiangsu, China) or intravenous iohexol (Omnipaque 300, GE Healthcare) or iodixanol (Visipaque 320, GE Healthcare) intravenously by means of a mechanical power injector (Stellant Injector System, Medrad Inc., Warrendale, PA, USA), administered at a rate of 3-4 mL/sec, followed by a 15- to 20$\mathrm{mL}$ saline flush. The standard protocol for triphasic CT consisted of an unenhanced, arterial phase with a scanning delay of 30-40 seconds, and a portal venous phase with a scanning delay of $60-80$ seconds.

\section{Interpretation of the Images}

The reference criterion for the diagnosis of fatty liver was the liver attenuation on the unenhanced CT. Hounsfield unit attenuation values of the liver and spleen were obtained through circular regions of interest representing the parenchyma on the unenhanced $\mathrm{CT}$ by a radiologist (R.T.), taking care to avoid visible vessels, bile ducts, focal lesions, calcifications, focal changes of fatty liver or fatty sparing, and the surface margins. The regions of interest were located in the right hepatic lobe and left hepatic lobe. The average of these two regions of attenuation was then obtained. The degree of the fatty liver was graded according to the criteria based on the ratio of the $\mathrm{CT}$ values of liver and spleen (L/S) proposed by Zeng et al. [11] 
in which $0.7<\mathrm{L} / \mathrm{S}$ ratio $<1.0,0.5<\mathrm{L} / \mathrm{S}$ ratio $\leq 0.7$, and $\mathrm{L} / \mathrm{S}$ ratio $\leq 0.5$ corresponded to mild, moderate, and severe fat content categories, respectively.

Taking the findings on $\mathrm{CT}$ as reference, the presence or absence of focal liver lesions was determined, findings on US were compared, and several characteristics of the focal lesions were evaluated.

\section{Statistical Analysis}

The continuous variables included the size of the focal liver lesions, age of the patients, etc.; the discrete variables included the US detection rate of focal liver lesions in patients with and without fatty liver. The continuous variables were expressed as mean \pm standard deviation, while variables of different groups were compared using the independent samples $t$ test. The non-continuous variables were analyzed using the chi-squared test. The statistical analyses were performed using IBM SPSS ver. 20 (IBM Co., Armonk, NY, USA). A P-value of less than 0.05 was considered to indicate a statistically significant difference.

\section{Results}

The number of patients with and without fatty liver, fatty liver grades, and lesions visible and invisible on US are listed in Table 1. The pathologic nature of the focal liver lesions based on histopathology (if available) and presumed diagnosis included cavernous hemangioma, 241; simple cyst, 107; metastatic tumor, 193; focal nodular hyperplasia, 5; adenoma, 2; hepatocellular carcinoma, 113; cholangiocarcinoma, 4; inflammatory pseudotumor,

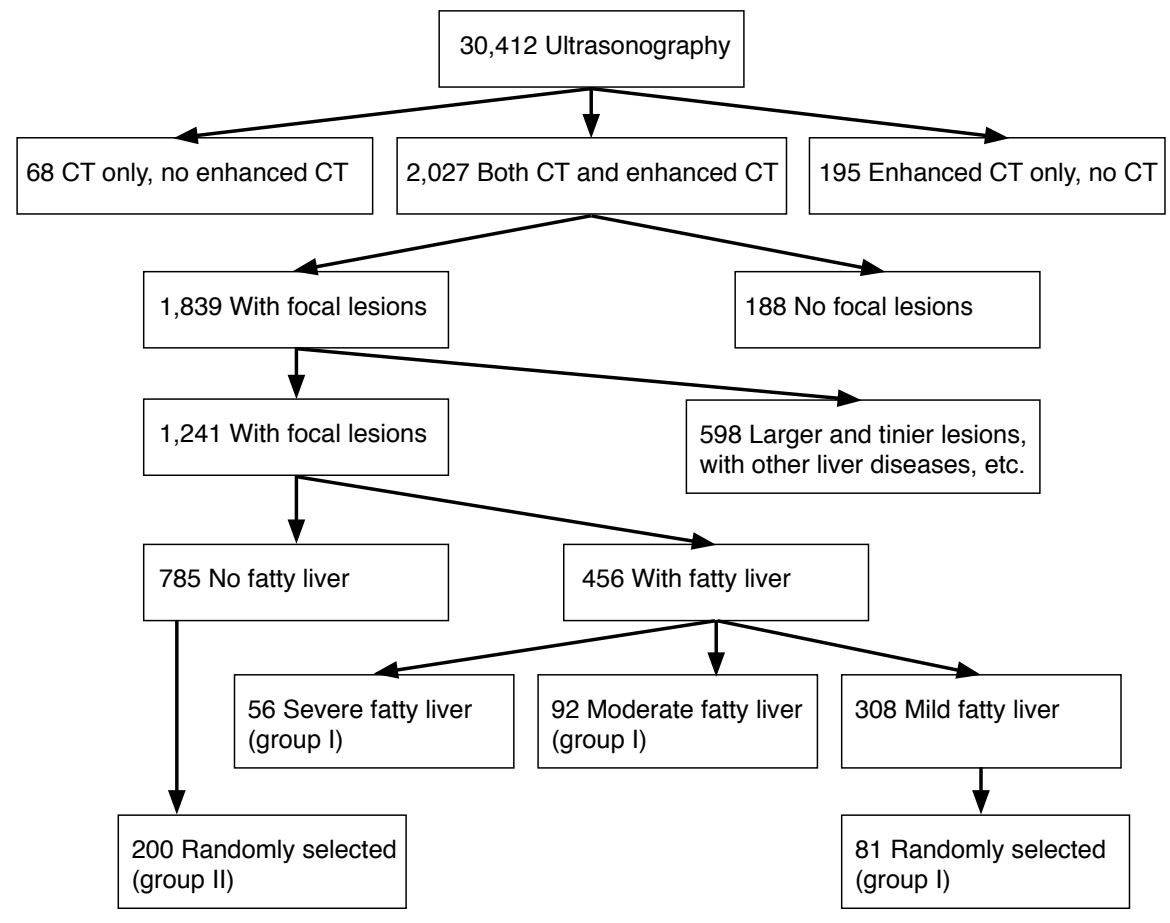

Fig. 1. A flow chart of study population selection. CT, computed tomography.

Table 1. Number of patients by presence and extent of fatty liver, and lesions visible and invisible on ultrasonography

\begin{tabular}{|c|c|c|c|c|}
\hline Patients and fatty liver grade & No. of lesions & Size of lesions $(\mathrm{mm})$ & Visible lesions, No. (\%) & Invisible lesions, No. (\%) \\
\hline Patients with fatty liver $(n=229)$ & 501 & $29.5 \pm 18.76$ & $435(86.8)$ & $66(13.2)$ \\
\hline Mild ( $n=81)$ & 177 & $30.0 \pm 18.93$ & $162(91.5)$ & $15(8.5)$ \\
\hline Moderate $(n=92)$ & 212 & $30.1 \pm 15.84$ & $190(89.6)$ & $22(10.4)$ \\
\hline Severe $(n=56)$ & 112 & $30.3 \pm 21.06$ & $83(74.1)$ & $29(25.9)$ \\
\hline Patients without fatty liver $(n=200)$ & 413 & $29.9 \pm 19.65$ & $389(94.2)$ & $24(5.8)$ \\
\hline P-value $e^{a)}$ & - & 0.329 & $<0.001$ & - \\
\hline
\end{tabular}

a) P-values: all 229 patients with fatty liver vs. all 200 patients without fatty liver. 
5; solitary necrotic nodule, 2; and undetermined lesions, 242. The number of focal liver lesions in groups I and II were 501 and 413, respectively. The US detection rates of focal liver lesions in patients with and without fatty liver were $86.8 \%$ (435/501) and 94.2\%

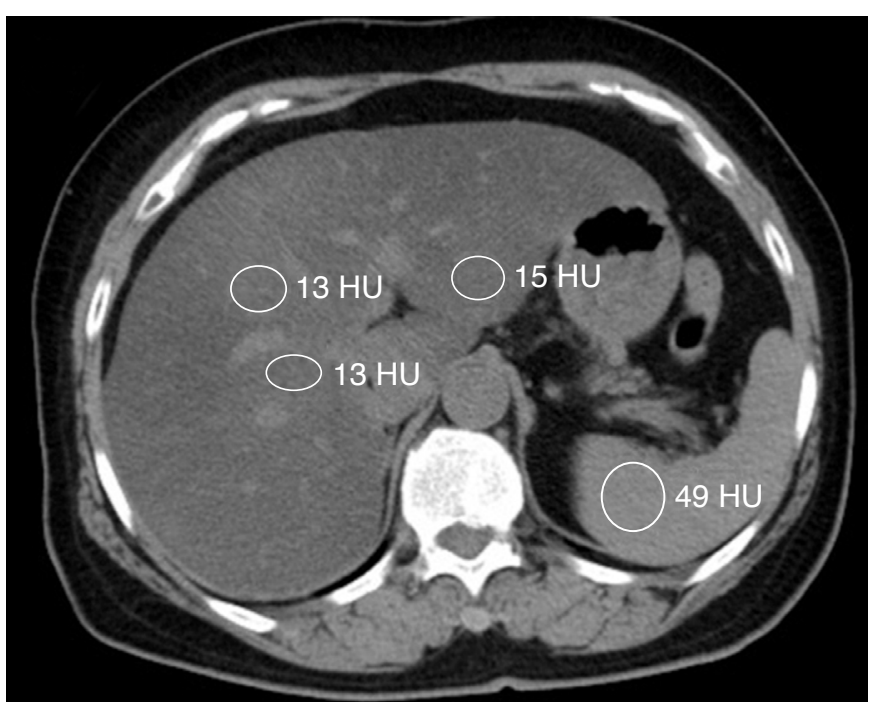

Fig. 2. Example of region of interest (ROI) placement for attenuation measurement on an unenhanced axial computed tomography image. Three ROls are placed in the liver at the level of the hepatic portal, and one is placed in the middle portion of the spleen. The liver measured $13 \mathrm{HU}$ and the spleen measured $49 \mathrm{HU}$, consistent with fatty infiltration of the liver. HU, Hounsfield unit.

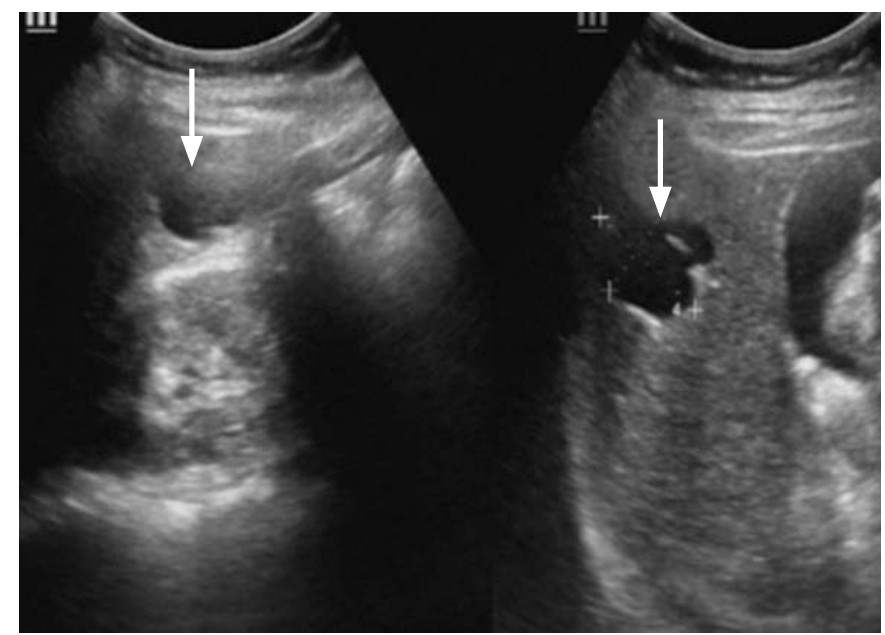

A

Fig. 3. A 37-year-old man with two hepatic hemangiomas (16-21 $\mathrm{mm}$ in diameter) and three cysts (15-22 $\mathrm{mm}$ in diameter), and whose liver does not have fat infiltration.

A. At ultrasonography examination, only liver cysts are detected (arrows), and no hemangioma is visualized. B. Axial contrast-enhanced computed tomography image obtained at the arterial phase shows two low-density lesions enhancing centripetally and slowly, while the three lower lesions were found to lack enhancement (not shown). The image obtained in the portal venous phase shows two low-density lesions enhancing centripetally (arrows), but the three lower lesions still did not have enhancement. The results suggest that the two low density lesions are hepatic hemangiomas, and the three unenhanced lesions are cysts. 


\section{Discussion}

For the focal lesion assessment in the fatty liver, many reports have noted the difficulty of image interpretation due to the change in the echo pattern of the focal lesions on a fatty background $[4,7,8,12]$. However, the performance of US in the detection of focal liver lesions in fatty liver has not yet been thoroughly addressed. In the present study, the detection rate of focal liver lesions on US in patients with severe fatty liver was significantly lower than in other patients.

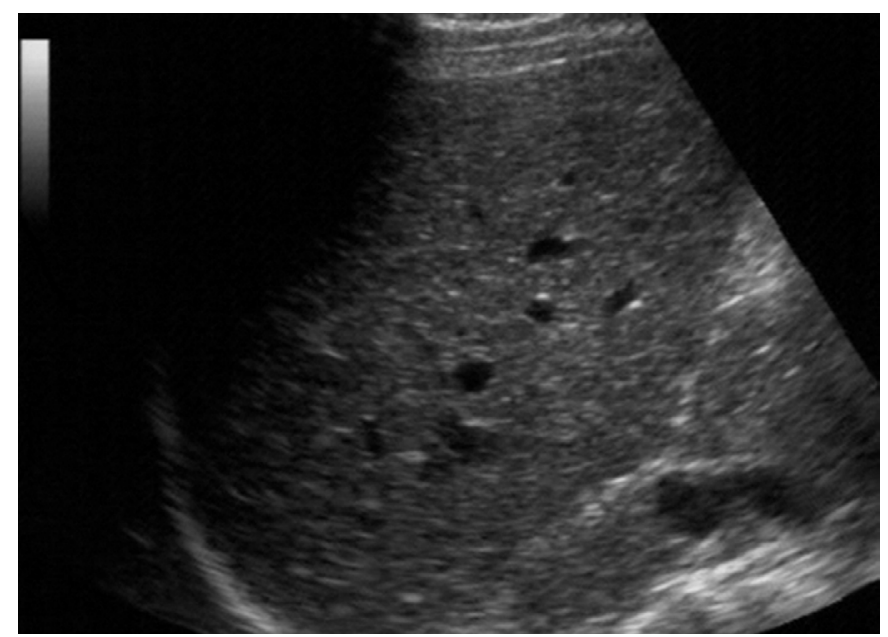

A

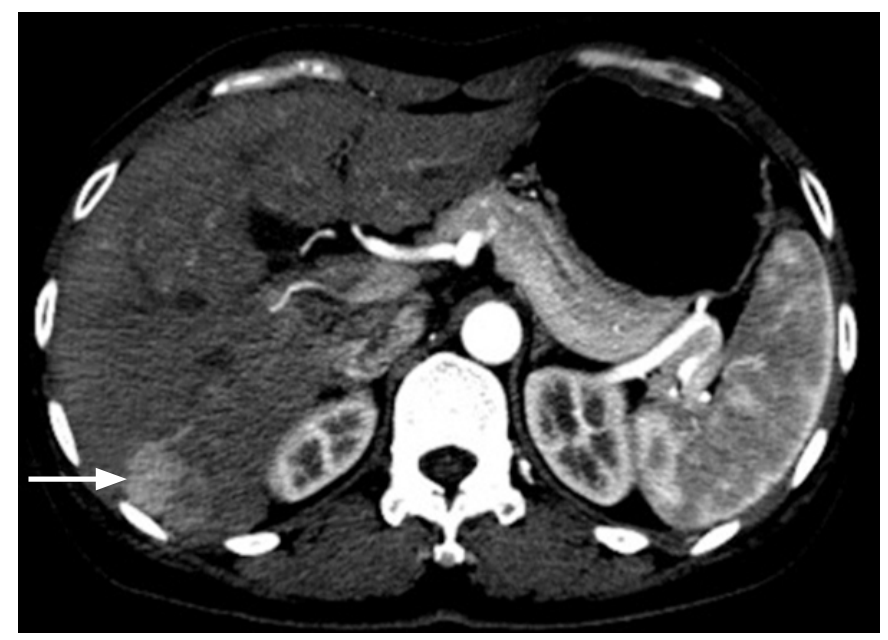

C
A study by van Vledder et al. [3] showed that the quality of US is poorer overall in the presence of fatty liver; some hypoechoic colorectal liver metastatic cancers become more conspicuous in fatty liver, and some hyperechoic or isoechoic lesions without accompanying fatty liver become less conspicuous in fatty liver. This indicates that some focal lesions become easier to visualize in the presence of fatty liver, but others are the opposite. The US visualization of a focal lesion might depend on both the intrinsic echogenicity of the lesion and the contrast between the lesion and the surrounding parenchyma, in conjunction with the size,

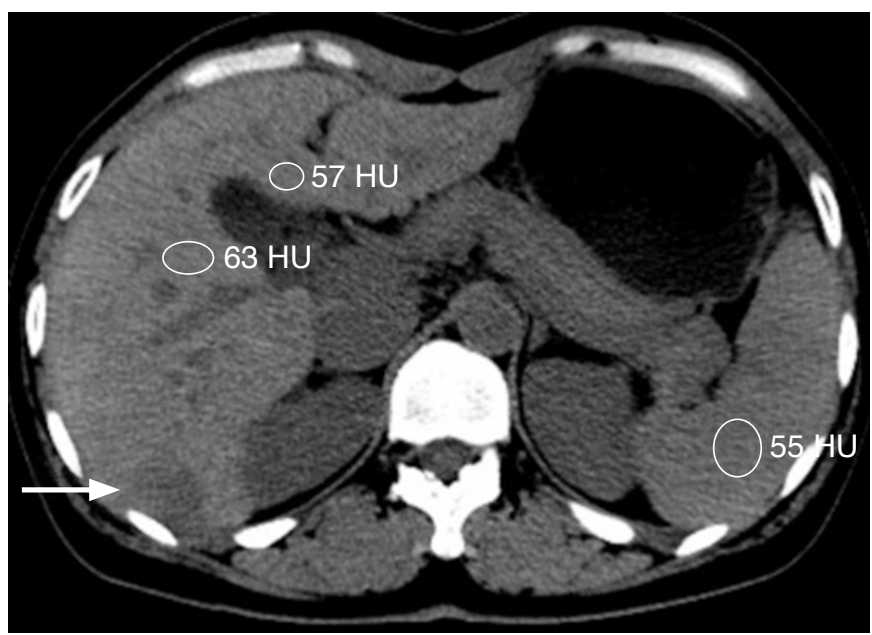

B

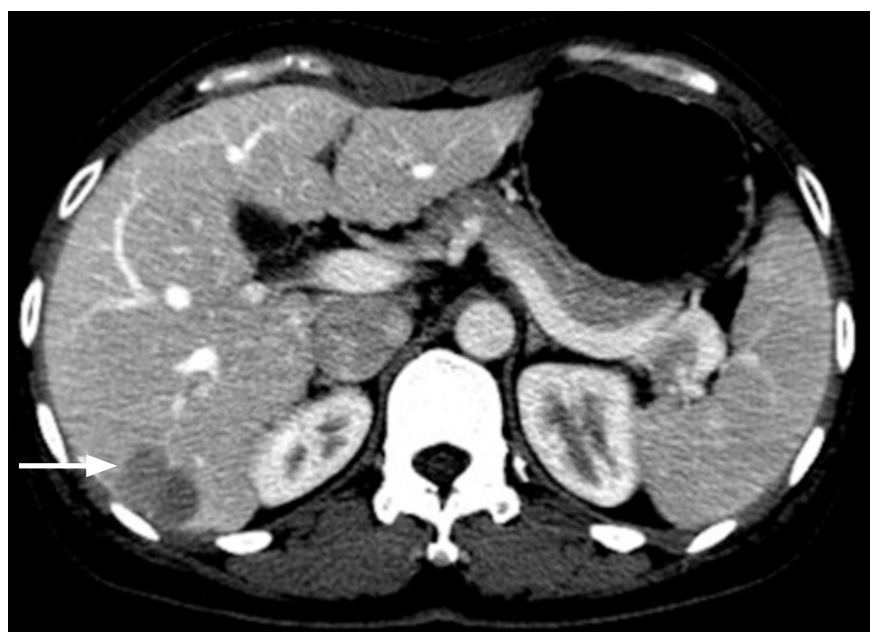

D

Fig. 4. A 54-year-old man with hepatocellular carcinoma (40 $\mathrm{mm}$ in diameter), and whose liver does not have fat infiltration, but with liver parenchyma that is coarse due to fibrosis.

A. At ultrasonography examination, no liver focal lesion is visualized. B. An axial unenhanced computed tomography (CT) image shows the presence of a liver focal lesion (arrow). The CT value of the liver is $63 \mathrm{HU}$ and spleen is $55 \mathrm{HU}$, which is consistent with liver without fatty infiltration. C. Axial contrast-enhanced CT image obtained at the arterial phase shows rapid enhancement of the lesion (arrow). D. Axial contrast-enhanced CT image obtained in the portal venous phase shows the quick "washout effect" of the lesion (arrow). The results indicate that the focal lesion is hepatocellular carcinoma. HU, Hounsfield unit. 


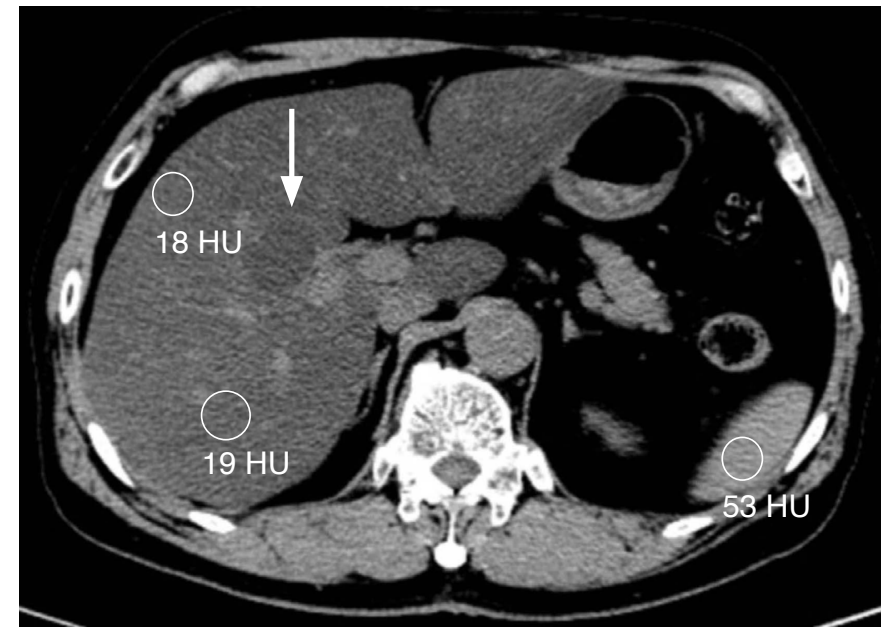

A

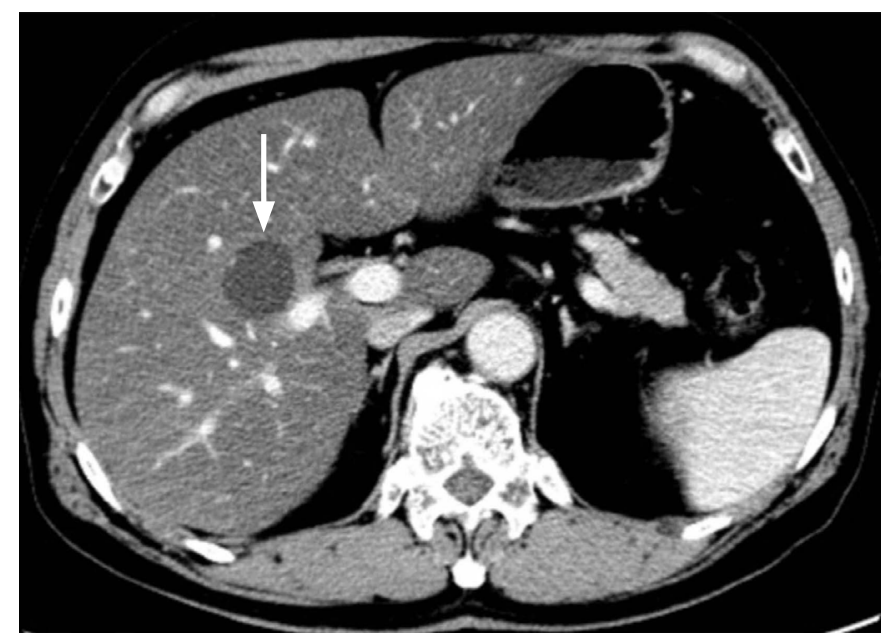

C

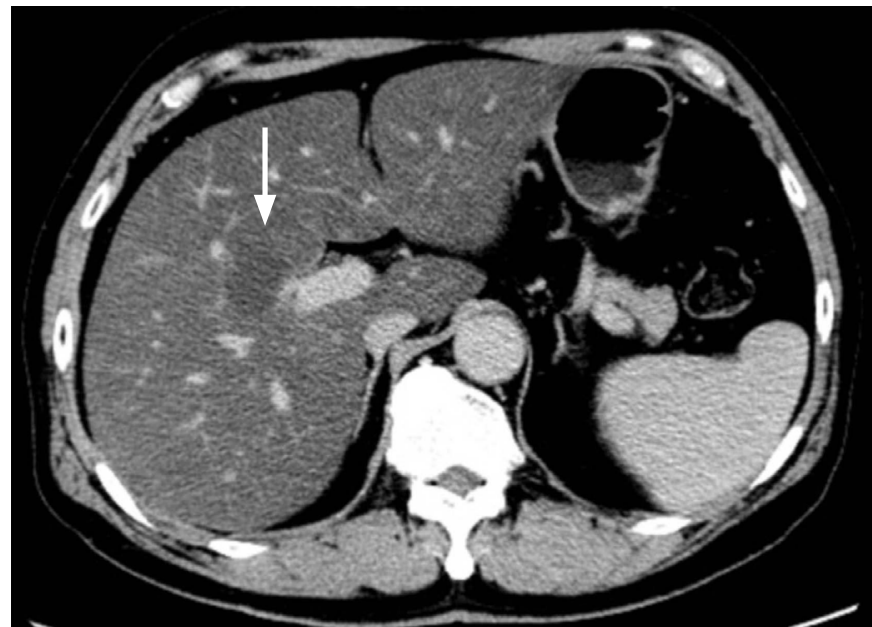

B

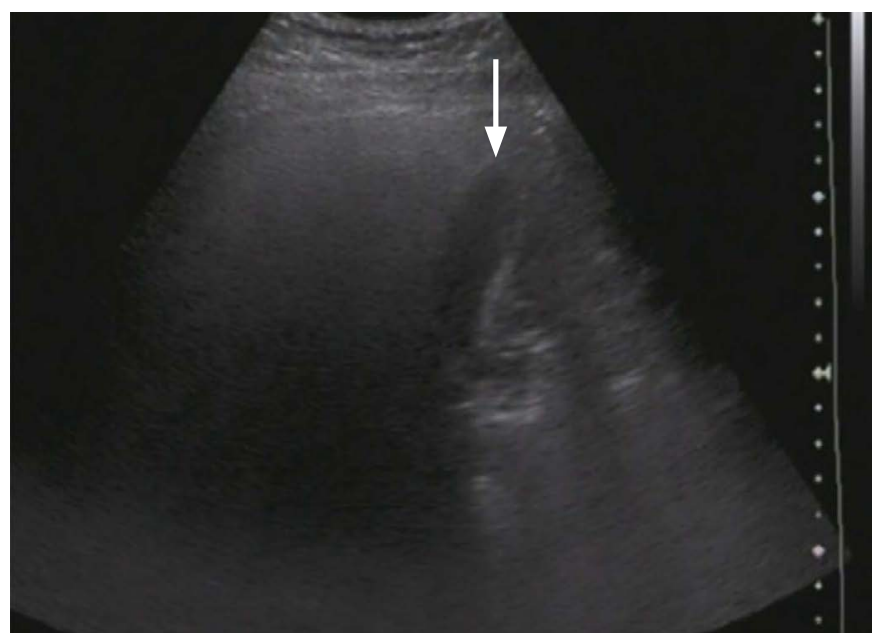

D

Fig. 5. A 29-year-old man with severe fatty liver and focal liver lesion (26 $\mathrm{mm}$ in diameter).

A. Axial unenhanced computed tomography (CT) image shows the lesion presenting slightly lower density (arrow) relative to the abutting parenchyma. The CT value of the liver is $18 \mathrm{HU}$ and that of the spleen is $53 \mathrm{HU}$, which is consistent with fatty infiltration of the liver. B. Axial image obtained in the arterial phase of enhanced CT shows the lesion has no enhancement (arrow). C. Axial image obtained in portal venous phase of enhanced CT shows the lesion has no enhancement (arrow). The results suggest the lesion is a cyst. D. Image in the transverse plane obtained by ultrasonography shows high echogenicity of the liver parenchyma and substantial attenuation; the gallbladder (arrow) is partially obscured, and the diaphragm and liver veins are almost invisible. No focal lesion is visible in the liver. HU, Hounsfield unit.

location, and depth of the lesion. Apart from the influence of the contrast in resolution between the focal lesion and the surrounding parenchyma with greater echogenicity, the presence of fatty liver would negatively affect the ultrasound beam penetration, resulting in a compromise of focal lesion detection. It is well known that hepatic veins appear thin or obscure in moderate and severe fatty liver. The reason for this phenomenon is not that the hepatic veins become thin or disappear, but the impact of the presence of fatty liver. With severe fatty liver, even the gallbladder wall becomes blurry when it is scanned axially from the intercostal region; yet, when scanned obliquely from the subcostal region, which keeps away the thick liver parenchyma, it appears distinct. Our study results seemed to be consistent with the fact that severe fat infiltration in the liver is known to cause acoustic attenuation and to obscure the visualization of the intrahepatic structures and focal lesions.

Teefey et al. [1] reported that small size was the most common cause of a missed diagnosis of focal liver lesion in US, CT, or MRI. In the present study, the detectability of the lesions varied significantly between the cases with fatty liver and those without fat infiltration, but the sizes of the invisible lesions in the two groups were not 
significantly different from each other. Moreover, the lesions invisible in our study were not the smallest ones or all smaller entities. This indicated that lesion size is not the dominant factor affecting the visualization of focal liver lesions in the presence of fat infiltration. In clinical practice, some tiny punctate calcifications in the liver can be detected without effort due to their high echogenicity, which has much higher contrast to the liver parenchyma than that of other lesions; furthermore, if any lesion is located in the surface of the liver, it should also be readily visible regardless of the lesion size. In terms of the relationship to fatty liver, lesion size should not have a notable effect on the detection rate of focal liver lesions.

The potential limitations of this study include the following: (1) Only those lesions frequently encountered in clinical practice, but not the less common larger and smaller lesions, were included, which may have resulted in sampling bias. On the other hand, the samples were acquired from groups of patients with and without fatty liver in the same way, and no significant difference in the lesion size was found between the two groups; therefore, the sampling bias is negligible. (2) No stratified analysis according to the size range of the lesions was performed, which may have affected the accuracy of analysis of the relationship to fatty liver. However, the results of this study show that the size of lesions invisible in the two groups was not significantly different, which indicates that the absence of size stratification had little effect on the analysis. (3) The correlation of histological findings was limited, and no data analysis was performed that distinguished between benign and malignant lesions. However, cystic lesions are easier to detect in the liver than solid lesions in general. In this study, the samples were selected by randomization, so bias caused by the lesion's pathology would have been limited. (4) No echoic texture analysis was performed for individual lesions, which may have affected the analysis in individual cases. However, the sample of this study was relatively large, and the impact of echoic texture is trivial.

In conclusion, the results of our study showed that a fatty liver background did not always influence the detection of focal lesions in the liver; specifically, mild and moderate fatty liver did not significantly affect the visualization of lesions, while severe fatty liver impaired the detection of focal lesions in the liver. Therefore, clinicians should be aware that if a patient with mild fatty liver is suspected of having a liver tumor, noninvasive US can be used primarily, but if a patient with severe fatty liver is suspected to have a liver tumor, US should only be used cautiously, given the potential for a missed diagnosis.

ORCID: Size Wu: http://orcid.org/0000-0002-1086-764X; Rong Tu: http://orcid.org/00000002-0030-4376; Ruixia Nan: http://orcid.org/0000-0002-9493-9638; Guangqing Liu: http://orcid.org/0000-0002-2745-7225; Xiaojing Cui: http://orcid.org/0000-0003-0330-
7772; Xian Liang: http://orcid.org/0000-0002-0175-3499

\section{Conflict of Interest}

No potential conflict of interest relevant to this article was reported.

\section{Acknowledgments}

The authors would like to express their gratitude to the sonologists, Li Huang, Ying Wan, Shibin Lin, Enhai Zheng, Chuan Fu, Kailiang Chen, and Yanyan Zhao for their assistance.

\section{References}

1. Teefey SA, Hildeboldt CC, Dehdashti F, Siegel BA, Peters MG, Heiken $J$, et al. Detection of primary hepatic malignancy in liver transplant candidates: prospective comparison of CT, MR imaging, US, and PET. Radiology 2003;226:533-542.

2. Liu WC, Lim JH, Park CK, Kim MJ, Kim SH, Lee SJ, et al. Poor sensitivity of sonography in detection of hepatocellular carcinoma in advanced liver cirrhosis: accuracy of pretransplantation sonography in 118 patients. Eur Radiol 2003;13:1693-1698.

3. van Vledder MG, Torbenson MS, Pawlik TM, Boctor EM, Hamper UM, Olino K, et al. The effect of steatosis on echogenicity of colorectal liver metastases on intraoperative ultrasonography. Arch Surg 2010;145:661-667.

4. Konno K, Ishida H, Sato M, Komatsuda T, Ishida J, Naganuma $\mathrm{H}$, et al. Liver tumors in fatty liver: difficulty in ultrasonographic interpretation. Abdom Imaging 2001;26:487-491.

5. Bennett GL, Krinsky GA, Abitbol RJ, Kim SY, Theise ND, Teperman LW. Sonographic detection of hepatocellular carcinoma and dysplastic nodules in cirrhosis: correlation of pretransplantation sonography and liver explant pathology in 200 patients. AJR Am J Roentgenol 2002;179:75-80.

6. Quaia E, D'Onofrio M, Palumbo A, Rossi S, Bruni S, Cova M. Comparison of contrast-enhanced ultrasonography versus baseline ultrasound and contrast-enhanced computed tomography in metastatic disease of the liver: diagnostic performance and confidence. Eur Radiol 2006;16:1599-1609.

7. Marsh JI, Gibney RG, Li DK. Hepatic hemangioma in the presence of fatty infiltration: an atypical sonographic appearance. Gastrointest Radiol 1989;14:262-264.

8. Vilgrain V, Ronot M, Abdel-Rehim M, Zappa M, d'Assignies G, Bruno O, et al. Hepatic steatosis: a major trap in liver imaging. Diagn Interv Imaging 2013;94:713-727.

9. Wong VW, Chu WC, Wong GL, Chan RS, Chim AM, Ong A, et al. Prevalence of non-alcoholic fatty liver disease and advanced fibrosis in Hong Kong Chinese: a population study using protonmagnetic resonance spectroscopy and transient elastography. Gut 2012:61:409-415.

10. Williams CD, Stengel J, Asike MI, Torres DM, Shaw J, Contreras M, et 
al. Prevalence of nonalcoholic fatty liver disease and nonalcoholic steatohepatitis among a largely middle-aged population utilizing ultrasound and liver biopsy: a prospective study. Gastroenterology 2011;140:124-131.

11. Zeng MD, Fan JG, Lu LG, Li YM, Chen CW, Wang BY, et al. Guidelines for the diagnosis and treatment of nonalcoholic fatty liver diseases. J Dig Dis 2008;9:108-112.

12. Soyer $P$, Sirol M, Fargeaudou $Y$, Duchat F, Hamzi L, Boudiaf $M$, et al. Differentiation between true focal liver lesions and pseudolesions in patients with fatty liver: evaluation of helical CT criteria. Eur Radiol 2010;20:1726-1737. 